\title{
Quantum mechanical effect of path-polarization contextuality for a single photon
}

\author{
Alok Kumar Pan* and Dipankar Home ${ }^{\dagger}$ \\ CAPSS, Department of Physics, Bose Institute, Sector-V, Salt Lake, Calcutta 700091, India
}

\begin{abstract}
Using measurements pertaining to a suitable Mach-Zehnder(MZ) type setup, a curious quantum mechanical effect of contextuality between the path and the polarization degrees of freedom of a polarized photon is demonstrated, without using any notion of realism or hidden variables - an effect that holds good for the product as well as the entangled states. This form of experimental context-dependence is manifested in a way such that at either of the two exit channels of the MZ setup used, the empirically verifiable subensemble statistical properties obtained by an arbitrary polarization measurement depend upon the choice of a commuting(comeasurable) path observable, while this effect disappears for the whole ensemble of photons emerging from the two exit channels of the MZ setup.
\end{abstract}

PACS numbers: 03.65.Ta

\section{INTRODUCTION}

Investigations of the implications of a possible 'incompleteness' of quantum mechanics(QM) have resulted in striking discoveries of fundamental constraints which any realist model has to satisfy in order to be compatible with the empirically verifiable predictions of QM. One of such constraints is, of course, the comprehensively studied incompatibility between QM and the local realist models of quantum phenomena discovered using Bell's theorem [1, 2] and its variants [3] - for a comparatively recent review of investigations in this area, see, for example, [4]. The other constraint that, of late, has also been attracting an increasing attention is the one concerning the inconsistency between QM and the noncontextual realist(NCR) models(the Bell-Kochen-Specker theorem [5, 6] and its variants[8-26]). It is this latter strand of study which leads to the present paper. For this, we proceed by first recapitulating the essence of what is usually meant by the notion of 'noncontextuality'.

Given any realist hidden variable model of quantum phenomena, the individual measured values of any dynamical variable are predetermined by the appropriate values of hidden variables $(\lambda$ 's) which are used in a realist model for a 'complete specification' of the state of an individual quantum system. Now, the condition of 'noncontextuality', in its most general form underlying its usual use, stipulates that the predetermined individual measured value of any dynamical variable, for a given $\lambda$, is the same whatever be the way the relevant dynamical variable is measured. The question as to what extent this putative condition is compatible with the formalism of QM has been subjected to two different lines of study by exploring the implications of two separate facets of this condition.

One of these is contingent upon assuming that the predetermined individual measured value of a given dynam-

\footnotetext{
*apan@bosemain.boseinst.ac.in

†dhome@bosemain.boseinst.ac.in
}

ical variable is independent of whatever be the choice of the other commuting(comeasurable) observable that is measured along with it. This condition has led to the formulation of a testable Bell-type inequality [13] that is derived as a consequence of the NCR models, but is violated by QM for the entangled states by a finite amount, thereby enabling an empirical discrimination between QM and the NCR models 12, 14, 15]. Subsequently, the experimental investigation along this line has been enriched by further studies [17].

The other line of study on the issue of contextuality visa-vis QM is based upon a feature characterising the NCR models that can be expressed as follows: For an individual measurement, the definite outcome obtained for an observable(say, $A$ ), as specified by a given hidden variable $\lambda$, be denoted by $v(A)$. Now, let $B$ be any other commuting(comeasurable)observable whose measured value in an individual measurement, as fixed by the same given $\lambda$, be denoted by $v(B)$. Then, if one denotes an individual outcome of a holistic measurement of the product observable $A B$ by $v(A B)$ which is determined by the same value of the hidden variable $\lambda$, the notion of noncontextuality is taken to imply the following condition(known as the 'product rule')

$$
v(A B)=v(A) v(B)
$$

which is assumed to hold good independent of the experimental procedure(context) used for measuring the joint observable $A B$ in a holistic way, and is also independent of the way the individual separate measurements of $A$ and $B$ are performed separately. This feature of noncontextuality was elegantly used by Mermin[7] for formulating a proof of quantum incompatibility with the NCR models for two spin- $1 / 2$ particles that holds for any state. Later, Cabello[22] cast Mermin's proof[7] in the form of a testable inequality involving the statistically measurable quantities - this inequality being violated by QM by a finite measurable amount for an arbitrary two-qubit state. Subsequently, the state-independent quantum violation of this inequality has been experimentally corroborated using the polarization and the linear momentum degrees of freedom of a single photon 23]. 
In contrast to the above two directions of study, in this paper we explore a third line of probing, initiated in a recent paper 26] that used a suitable path-spin entangled state of a spin- $1 / 2$ particle, in which the issue of contextuality is probed within the framework of QM, devoid of any reference to the NCR models. With a view of extending the ambit of this new line of study, the present paper reveals that that it is indeed possible to show a form of state-independent contextuality within QM for any state that is an entangled or a product state in the four dimensional space. By using photons and an appropriate setup we show that a statistically discernible effect of the path-polarization interdependence is manifested in terms of the operationally suitably defined subensemble statistical properties of an arbitrary polarization measurement that depend upon the choice of a comeasurable(commuting)path observable. Interestingly, this context-dependence gets obliterated for the statistical results pertaining to the whole ensemble of photons emerging from the setup used for the polarization measurement, whatever be the choice of the comeasurable path observable. Let us now proceed by first explaining the specifics of the setup(Figure 1) that is required for our demonstration.

\section{THE SETUP FOR PREPARING THE REQUIRED PRODUCT $O R$ AN ENTANGLED STATE}

In order to formulate our argument, the required pathpolarization product state can be prepared using a 50:50 beam splitter(BS1), while an entangled path-polarization state can be prepared by using a 50:50 BS1 in conjunction with a half wave plate(HWP) that is placed in one of the output ports of the BS1(Fig.1). The relevant path and the polarization measurements will be considered separately for these prepared states.

Let us consider that an ensemble of photons having horizontal polarized state $|H\rangle$ be incident on a 50:50 beam-splitter(BS1). Any given incident photon can then emerge along either the reflected or the transmitted channel corresponding to the state designated by $|r\rangle$ or $|t\rangle$ respectively. Here note that for any given lossless beamsplitter, arguments using the unitarity condition show that for the photons incident on BS1, the phase shift between the transmitted and the reflected states of photons is essentially $\pi / 2[27]$. The prepared path-polarization product state after emerging from BS1 can then be written as

$$
|\Psi\rangle_{p r}=\frac{1}{\sqrt{2}}(|t\rangle+i|r\rangle)|H\rangle
$$

On the other hand, for preparing an entangled pathpolarization state, photons in the channel corresponding to $|t\rangle$ are passed through a HWP that flips the polarization $|H\rangle$ to $|V\rangle$. The state emerging from HWP can then be written as

$$
|\Psi\rangle_{e n}=\frac{1}{\sqrt{2}}(|t\rangle|V\rangle+i|r\rangle|H\rangle)
$$

which is an entangled state between the path and the polarization degrees of freedom.

In writing both Eqs.(2) and (3) we have taken into account a relative phase shift of $\pi / 2$ between the states $|r\rangle$ and $|t\rangle$ that arises because of the reflection from BS1. Eqs.(2) and (3) represent the prepared states on which we will separately consider the relevant path and the polarization measurements for the purpose of showing the path-polarization interdependence for the product as well as for the entangled state. For this, we proceed as follows.

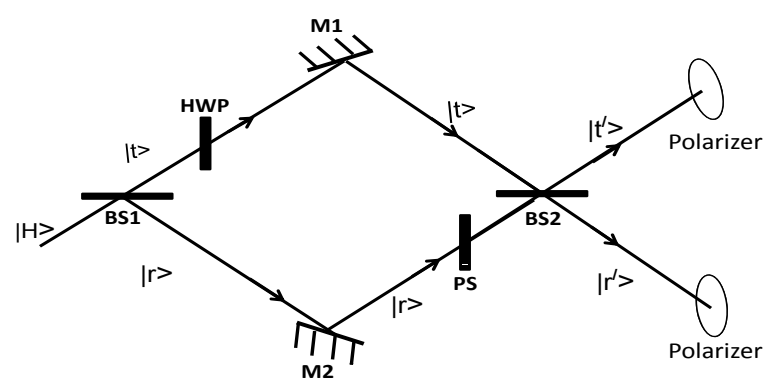

Figure 1: Horizontally polarized (denoted by $|H\rangle)$ photons enter the indicated Mach-Zehnder type setup through a 50:50 beamsplitter BS1, and pass through the channels corresponding to $|t\rangle$ and $|r\rangle$ thereby generating the path-polarization product state. For generating an entangled path-polarization state, a half wave plate (HWP) that flips the horizontally polarized state $|H\rangle$ into the vertically polarized state $|V\rangle$ is placed along one of the channels $|t\rangle$. Subsequently, for the required measurements pertaining to the path observable, a phase-shifter(PS) is placed along the channel $|r\rangle$ that creates a relative phase shift between the channels $|t\rangle$ and $|r\rangle$. The two channels are then recombined at a 50:50 beamsplitter BS2. The PS in conjunction with BS2 serves the purpose of path measurement(see the text). Finally, the measurement in an arbitrary polarization basis is made by using two polarizers that are placed along the two output channels $\left|t^{\prime}\right\rangle$ and $\left|r^{\prime}\right\rangle$ of the BS2. The path-polarization interdependence can then be demonstrated by considering the subensemble mean values associated with each of the two output channels $\left|t^{\prime}\right\rangle$ and $\left|r^{\prime}\right\rangle$.

\section{THE QM DEMONSTRATION OF PATH-POLARIZATION INTERDEPENDENCE}

After passing through the mirrors M1 and M2, photons are subjected to a phase shifter(PS) along the channel $|r\rangle$ which introduces a relative phase shift of $\phi$ between the path channels $|r\rangle$ and $|t\rangle$. The two path states are then recombined at BS2, and the output path states $\left|t^{\prime}\right\rangle$ and 
$\left|r^{\prime}\right\rangle$ are respectively given by

$$
\begin{aligned}
\left|t^{\prime}\right\rangle & =\frac{1}{\sqrt{2}}\left(e^{i \phi}|r\rangle-i|t\rangle\right) \\
\left|r^{\prime}\right\rangle & =\frac{1}{\sqrt{2}}\left(-i e^{i \phi}|r\rangle+|t\rangle\right)
\end{aligned}
$$

Eqs.4a 4b show that, for a given linear combination of $|t\rangle$ and $|r\rangle$, using the different values of $\phi$, one can unitarily generate at the output of BS2 various linear combinations of $|t\rangle$ and $|r\rangle$ that correspond to different probability amplitudes of finding photons in the channels corresponding to $\left|t^{\prime}\right\rangle$ and $\left|r^{\prime}\right\rangle$. This, in turn, implies that PS in conjunction with BS2 can be regarded as corresponding to different choices of the path observables $\widehat{\beta}_{\phi}=\left|t^{\prime}\right\rangle\left\langle t^{\prime}|-| r^{\prime}\right\rangle\left\langle r^{\prime}\right|$ with eigenvalues \pm 1 . Such observables, in terms of actual measurements, correspond to different relative counts registered by the detectors placed along the channels represented by $\left|t^{\prime}\right\rangle$ and $\left|r^{\prime}\right\rangle$. Using Eqs. (4a 4b) the path observable is of the form

$$
\beta_{\phi}=\left(\begin{array}{cc}
0 & i e^{i \phi} \\
-i e^{-i \phi} & 0
\end{array}\right)
$$

that can be written as the following linear combination of the Pauli matrices

$$
\beta_{\phi}=-\sin \phi \widehat{\sigma}_{x}+\cos \phi \widehat{\sigma}_{y}=\vec{\sigma} \cdot \vec{n}_{\phi}
$$

where $\vec{n}_{\phi}=-\sin \phi \widehat{i}+\cos \phi \widehat{j}$.

It is, therefore, evident from Eq.(5) that the path observable $\widehat{\beta}_{\phi}$ which is represented by the vector component given by Eq.(6) varies according to the magnitude of the phase shift $\phi$, i.e., different choices of $\phi$ provide different contexts pertaining to the polarization measurements.

Next, we consider the measurement of an arbitrarily chosen polarization variable, say, $\widehat{\delta}$ which is given by

$$
\widehat{\delta}=\left|H^{\prime}\right\rangle\left\langle H^{\prime}|-| V^{\prime}\right\rangle\left\langle V^{\prime}\right|
$$

whose eigenstates are $\left|H^{\prime}\right\rangle=\cos \alpha|H\rangle+\sin \alpha|V\rangle$ and $\left|V^{\prime}\right\rangle=\sin \alpha|H\rangle-\cos \alpha|V\rangle$, where $\alpha$ denotes the orientation of the two polarizers that are placed separately along the channels $\left|t^{\prime}\right\rangle$ and $\left|r^{\prime}\right\rangle$.

Now, we will consider the expectation value of the polarization observable $\widehat{\delta}$ that involves contributions from both the output subensembles corresponding to polarizers separately placed along the channels $\left|t^{\prime}\right\rangle$ and $\left|r^{\prime}\right\rangle$. The subensemble mean values of $\widehat{\delta}$ measured in each of the two output channels, calculated using either the prepared product or an entangled path-polarization state given by Eqs. $(2)$ and (3) respectively, are denoted by $(\bar{\delta})_{t^{\prime}}$ and $(\bar{\delta})_{r^{\prime}}$, whence

$$
\langle\widehat{\delta}\rangle_{\Psi_{p r / e n}}=(\bar{\delta})_{t^{\prime}}+(\bar{\delta})_{r^{\prime}}
$$

where the subscript $\Psi_{p r / e n}$ represents the prepared entangled or product states given by Eq.(2) or (3) respectively.
Note that all the three quantities occurring in the equality given by Eq.(8) have the same operational status as far as their statistical reproducibility is concerned. But there is a crucial distinction between the left and the right hand sides of Eq.(8) with respect to the issue of path-polarization interdependence. The quantity on the left hand side of Eq. (8), the expectation value of $\langle\widehat{\delta}\rangle_{\Psi_{p r / e n}}$ pertaining to the whole ensemble, is independent of which path observable is measured along with it. On the other hand, each of the quantities on the right hand side of Eq.(8), the subensemble mean values denoted by $(\bar{\delta})_{t^{\prime}}$ and $(\bar{\delta})_{r^{\prime}}$ are contingent upon the choice of the comeasurable path observable. This can be seen by considering the polarization measurement outcomes relevant to any one of the two output subensembles.

In order to display the manifestation of this form of context-dependence within QM, we consider two different experiments involving measurements of the path observable $\widehat{\beta}_{\phi}$ and the polarization variable $\widehat{\delta}$, first for the prepared product state given by Eq.(2), and then for the prepared entangled state given by Eq.(3).

\section{A. Path-polarization context-dependence for a product state}

We first consider the path-polarization product state given by Eq.(2) as the input state for which the state that emerges from BS2 can be written as

$$
|\Phi\rangle_{p r}=\frac{1}{2}\left[i\left|t^{\prime}\right\rangle\left(1+e^{i \phi}\right)+\left|r^{\prime}\right\rangle\left(1-e^{i \phi}\right)\right]|H\rangle
$$

Then it follows that the expectation value of the polarization observable $\widehat{\delta}$ pertaining to the whole ensemble of photons emerging from BS2 is of the form

$$
\langle\widehat{\delta}\rangle_{\Psi_{p r}}=\cos 2 \alpha
$$

which comprises the respective subensemble polarization mean values calculated from Eq.(9) given by

$$
(\bar{\delta})_{t^{\prime}}=\frac{(1+\cos \phi) \cos 2 \alpha}{2} ; \quad(\bar{\delta})_{r^{\prime}}=\frac{(1-\cos \phi) \cos 2 \alpha}{2}
$$

Next, we come to the crux of our argument indicated as follows that hinges on two different choices of $\phi$, and where the superscript $\beta_{0}\left(\beta_{\pi / 2}\right)$ is used to denote the choice of the path observable specifying the given context:

(a) Taking $\phi=0$, this implies the choice of a particular path observable $\widehat{\beta}_{0}=\vec{\sigma} \cdot \vec{n}_{0}$ where $\vec{n}_{0}=\widehat{j}$. In this case, using Eq.(11), we obtain

$$
(\bar{\delta})_{t^{\prime}}^{\left(\beta_{0}\right)}=\cos 2 \alpha ; \quad(\bar{\delta})_{r^{\prime}}^{\left(\beta_{0}\right)}=0
$$

while, using Eq.(2), the polarization expectation value for the whole ensemble, $\langle\widehat{\delta}\rangle_{\Psi_{p r}}=\cos 2 \alpha$. 
(b) Taking $\phi=\pi / 2$, this implies the choice of a different path observable $\widehat{\beta}_{\pi}=\vec{\sigma} \cdot \vec{n}_{\pi / 2}$ where $\vec{n}_{\pi / 2}=-\widehat{i}$. Consequently, using Eq.(11), we obtain

$$
(\bar{\delta})_{t^{\prime}}^{\left(\beta_{\pi / 2}\right)}=\frac{\cos 2 \alpha}{2} ; \quad(\bar{\delta})_{r^{\prime}}^{\left(\beta_{\pi / 2}\right)}=\frac{\cos 2 \alpha}{2}
$$

while, using Eq.(2), the polarization expectation value for the whole ensemble remains the same, $\langle\widehat{\delta}\rangle_{\Psi_{p r}}=\cos 2 \alpha$.

It is then evident from Eqs.(10-13) that, while the quantum expectation value $\langle\widehat{\delta}\rangle_{\Psi_{p r}}$ of the observable $\widehat{\delta}$ pertaining to the whole ensemble remains the same for both the choices of $\widehat{\beta}_{0}$ and $\widehat{\beta}_{\pi / 2}$, the path-polarization context-dependence gets manifested in terms of the subensemble polarization mean values given by the testable quantities $(\bar{\delta})_{t^{\prime}}^{\left(\beta_{0}, \beta_{\pi / 2}\right)}$ and $(\bar{\delta})_{r^{\prime}}^{\left(\beta_{0}, \beta_{\pi / 2}\right)}$. To put it precisely, in our example, the interdependence between the path and the polarization degrees of freedom has the following operational meaning

$$
(\bar{\delta})_{t^{\prime}}^{\left(\beta_{0}\right)} \neq(\bar{\delta})_{t^{\prime}}^{\left(\beta_{\pi / 2}\right)} ; \quad(\bar{\delta})_{r^{\prime}}^{\left(\beta_{0}\right)} \neq(\bar{\delta})_{r^{\prime}}^{\left(\beta_{\pi / 2}\right)}
$$

i.e., the subensemble mean value of the polarization variable $\widehat{\delta}$ depends upon which of the path observables $\widehat{\beta}_{0}$ or $\widehat{\beta}_{\pi / 2}$ is comeasured, where both $\widehat{\beta}_{0}$ and $\widehat{\beta}_{\pi / 2}$ commute with $\widehat{\delta}$.

\section{B. Path-polarization context-dependence for an entangled state}

Now, we consider the path-polarization entangled state given by Eq.(3) as the input state for which the state that emerges from BS2 can be written as

$\left.|\Phi\rangle_{e n}=\frac{1}{2}\left[i\left|t^{\prime}\right\rangle\left(|V\rangle+e^{i \phi}|H\rangle\right)+\left|r^{\prime}\right\rangle\left(|V\rangle-e^{i \phi}|H\rangle\right)\right] 15\right)$

It follows from Eq.(15) that corresponding to the prepared path-polarization entangled state $|\Psi\rangle_{\text {en }}$ given by Eq.(3), the expectation value of the polarization variable $\widehat{\delta}$ for the whole ensemble of photons emerging from the beam-splitter BS2 is given by

$$
\langle\widehat{\delta}\rangle_{\Psi_{e n}}=0
$$

which is made up of the respective subensemble polarization mean values calculated from Eq.(15), which are of the form

$$
(\bar{\delta})_{t^{\prime}}=\frac{\sin 2 \alpha \cos \phi}{2} ; \quad(\bar{\delta})_{r^{\prime}}=-\frac{\sin 2 \alpha \cos \phi}{2}
$$

Then, similar to the argument given above for the input product state, in this case too Eq.(14) holds good, i.e., the path-polarization context-dependence gets manifested in terms of the subensemble polarization mean values given by the testable quantities $(\bar{\delta})_{t^{\prime}}^{\left(\beta_{0}, \beta_{\pi / 2}\right)}$ and $(\bar{\delta})_{t^{\prime}}^{\left(\beta_{0}, \beta_{\pi / 2}\right)}$, while the quantum expectation value $\langle\widehat{\delta}\rangle_{\Psi_{e n}}$ pertaining to the whole ensemble remains the same for both the choices of $\widehat{\beta}_{0}$ and $\widehat{\beta}_{\pi / 2}$.

\section{THE SIGNIFICANCE AND OUTLOOK}

The essence of what is demonstrated in this paper is as follows. A statistically discernible signature of interdependence between the path and the polarization degrees of freedom of polarized photons is revealed - an effect which is quantum mechanically calculable in terms of the measured values of a polarization variable pertaining to the operationally well-defined subensembles that comprise the final output ensemble at the two exit channels of our setup. The subensemble polarization mean value registered at either of the two exit channels varies according to the choice of the comeasurable(commuting) path observable(whose choice is fixed by the magnitude of the phase shift that is introduced by PS in the channel $|r\rangle)$ But, importantly, such a variation takes place by preserving the context-independence of the polarization expectation value that is defined for the whole output ensemble.

In other words, we show that for an arbitrarily prepared state that can be either a path-polarization entangled or a product state, a form of 'parameter dependence' is displayed in this example in a way that is restricted to the subensemble statistics. This is quite distinct from the issue of 'parameter independence' [33] for the EPRBohm type states involving the polarization variables of two spatially separated photons where the "parameter independence' is in the sense that no statistically discernible effect in any form can be detected in any one of the two wings of the EPR-Bohm pair that depends upon the measurement setting in the other wing - a possibility which is ruled out by the much-discussed 'no-signaling' condition 34]. But, in contrast, in our example, for an arbitrary input state considered in our example for a polarized photon, there is no such constraint which forbids the statistical manifestation of an intraparticle pathpolarization context-dependence. While such an effect does occur in our example, curiously, it is confined to the subensemble statistics in such a way that the "pathpolarization interdependence' disappears for the statistics of the whole ensemble.

In terms of the two distinct aspects of the NCR models as discussed earlier, we may recall that the statistically verifiable inequalities for the entangled as well as the product states have been analyzed, both theoretically and experimentally, thereby highlighting an incompatibility between QM and the NCR models. However, it needs to be stressed that these earlier demonstrations necessarily involve the assumption of the notion of real- 
ism that is used in tandem with the feature of noncontextuality at the level of individual measurement outcomes. In contrast, the statistically verifiable manifestation of the quantum mechanical 'path-polarization interdependence' shown in this paper is entirely independent of any notion of 'realism' or 'hidden-variables', because this effect is demonstrated essentially within the ambit of QM and, crucially, is independent of the nature of the input state which can be either a path-polarization product or an entangled state. A significant point to stress here is that a similar effect of interdependence showing a nonlocal connection between the entangled variables of the spatially separated photons cannot be demonstrated within QM unless one takes recourse to the notion of 'realism' or 'hidden-variables'. This fundamental distinction between quantum nonlocality and contextuality brought out by the example analyzed in this paper as well as in an ear- lier work 26] call for careful probing. Such investigations may provide useful insights into the type of constraints that would restrict the nonlocal realist models in the light of the recent studies 35 37] based on Leggett's work [38].

\section{Acknowledgements}

Authors thanks H. Rauch and Y. Hasegawa for the useful interactions that served as a prelude to this work. AKP recalls the discussions, in particular, with $\mathrm{A}$. Cabello during his visit to Benasque Center for Science, Spain. AKP acknowledges the Research Associateship of Bose Institute, Kolkata. DH acknowledges the project funding from DST, Govt. of India and support from Center for Science and Consciousness, Kolkata.
[1] Bell J S 1964 Physics, 1195

Clauser J F, Horne M A, Shimony A and Holt R A 1969 Phys. Rev. Lett. 23880

[2] Aspect A, Grangier P and Roger G 1981 Phys. Rev. Lett. 47460

Aspect A, Grangier P and Roger G 1981 Phys. Rev. Lett. 4991

[3] Greenberger D M, Horne M A, Shimony A and Zeilinger A,1990 Am. J. Phys. 581131

Hardy L 1993 Phys. Rev. Lett. 711665

[4] Genovese M 2005 Phys. Rep. 413319

[5] Bell J S 1966 Rev. Mod. Phys. 38447

[6] Kochen S and Specker E P 1967J. Math. Mech. 1759

[7] Mermin N D 1990 Phys. Rev. Lett. 653373 Mermin N D 1993 Rev. Mod. Phys. 65803

[8] Peres A 1990 Phys. Lett. A 151107

Peres A 1991 J. Phys. A 24, L175

Pertes A 1993 Quantum Theory: Concepts and Methods (Kluwer, Dordrecht) pp. 196-201

Kernaghan M and Peres A 1995 Phys. Lett. A 1981

[9] Cabello A, and Garcia-Alcaine G 1996 J. Phys. A 29 1025

Cabello A, Estebaranz J M and Garcia-Alcaine G 1996 Phys. Lett. A 212183

Cabello A, and Garcia-Alcaine G 1998 Phys. Rev. Lett. 801797

[10] Penrose R 1994 in Quantum Reflections edited by Ellis J and Amati A (Cambridge University Press, Cambridge)

[11] Roy S M and Singh V 1993 Phys. Rev. A 483379

[12] Simon C, Zukowski M, Weinfurter H, and Zeilinger A 2000 Phys. Rev. Lett. 851783

[13] Basu S, Bandyopadhyay S, Kar G, and Home D 2001 Phys. Lett. A 279281

[14] Michler M, Weinfurter H and Zukowski M 2000 Phys. Rev. Lett. 845457

[15] Hasegawa Y, Loidl R, Badurek G, Baron M and Rauch H 2003 Nature 42545

Hasegawa Y, Loidl R, Badurek G, Baron M and Rauch H 2004 J. Opt. B. 6 S7

[16] Huang Y F et al. 2003 Phys. Rev. Lett. 90250401
[17] Hasegawa Y et al. 2006 Phys. Rev. Lett. 97230401

[18] De Zela F 2007 Phys. Rev. A 76042119

[19] Cabello A, Filipp S, Rauch H and Hasegawa Y 2008 Phys. Rev. Lett. 100130404

[20] Klyachko A A, Can M A, Binicioglu S, and Shumovsky A S 2008 Phys. Rev. Lett. 101020403

[21] Bartosik H et al. 2009 Phys. Rev. Lett. 103040403

[22] Cabello A 2008 Phys. Rev. Lett. 101210401

[23] Kirchmair G et. al 2009 Nature 460494

[24] E. Amselem, M. Radmark, M. Bourennane and A. Cabello, Phys. Rev. Lett. 103, 160405 (2009)

[25] Gadway B R, Galvez E J and De Zela F 2009 J. Phys. B: At. Mol. Opt. Phys. 42015503

[26] Pan A K and Home D 2009 Phys. Lett. A 3733430

[27] Zeilinger A 1981 Am. J. Phys. 49882 Holbrow C H, Galvez E and Parks M E 2001 Am. J. Phys. $\mathbf{7 0 2 6 0}$

[28] Rosenfeld et al. 2009 Adv.Sci. Lett. 2469

[29] Moehring D L 2003 Phys. Rev. Lett. 93090410

[30] Ionicioiu R et al. 2005 Phys. Rev. A 63050101

[31] Ansmann M et al. 2009 Nature 461504

[32] Genovese M et al. 2001 Phys. Lett. B 513401

[33] Shimony A 1984 in Proceedings of the 1st International Symposium on the Foundations of Quantum Mechanics in the Light of New Technology edited by Kamefuchi S et al. (Physical Society of Japan, Tokyo) pp. 225-30 Jarrett J P 1984 Nous 18569

Jarrett J P 1989 in Philosophical Consequences of Quantum Theory edited by Cushing $\mathrm{J} \mathrm{T}$ and McMullin E(University of Norte Dame Press, Indiana) pp.60-79.

[34] Bohm D 1952 Quantum Theory (Englewood Cliffs, Prentice-Hall) pp.618-619

Ghirardi G C, Rimini A and Weber T 1980 Lett. Nuovo Cim. 27293

Eberhard P H and Ross R 1989 Found. Phys. Lett. 2127

[35] Groeblacher S et al. 2007 Nature 446871

[36] Branciard C et al. 2007 Phys. Rev. Lett. 99210407

[37] Laudisa F 2008 Found. Phys. 381110

[38] Leggett A J 2003 Found. Phys. 331469 